\title{
Direct measurement of $B\left(D_{s}^{+} \rightarrow \phi X^{+}\right)$
}

J. Z. Bai, ${ }^{1}$ O. Bardon, ${ }^{5, *}$ I. Blum, ${ }^{10}$ A. Breakstone,${ }^{8}$ T. Burnett, ${ }^{11}$ G. P. Chen,${ }^{1}$ H. F. Chen,${ }^{3}$ J. Chen, ${ }^{4}$ S. J. Chen, ${ }^{1}$ S. M. Chen, ${ }^{1}$ Y. Chen, ${ }^{1}$ Y. B. Chen, ${ }^{1}$ Y. Q. Chen, ${ }^{1}$ B. S. Cheng, ${ }^{1}$ R. F. Cowan,${ }^{5}$ X. Z. Cui, ${ }^{1}$ H. L. Ding, ${ }^{1}$ Z. Z. Du, ${ }^{1}$ W. Dunwoodie, ${ }^{7}$ X. L. Fan, ${ }^{1}$ J. Fang, ${ }^{1}$ C. S. Gao,${ }^{1}$ M. L. Gao, ${ }^{1}$ S. Q. Gao, ${ }^{1}$ P. Gratton, ${ }^{10}$ J. H. Gu, ${ }^{1}$ S. D. Gu, ${ }^{1}$ W. X. Gu, ${ }^{1}$ Y. F. Gu, ${ }^{1}$ Y. N. Guo,${ }^{1}$ S. W. Han,${ }^{1}$ Y. Han, ${ }^{1}$ F. A. Harris, ${ }^{8}$ J. He, ${ }^{1}$ M. He,${ }^{6}$ D. G. Hitlin, ${ }^{2}$ G. Y. Hu, ${ }^{1}$ T. Hu, ${ }^{1}$

X. Q. Hu, ${ }^{1}$ D. Q. Huang, ${ }^{1}{ }^{1}$ Y. Z. Huang, ${ }^{1}$ J. M. Izen, ${ }^{10}$ C. H. Jiang, ${ }^{1}$ S. Jin, ${ }^{1}$ Y. Jin,,${ }^{1}$ L. Jones,${ }^{2}$ S. H. Kang, ${ }^{1}$ Z. J. Ke, ${ }^{1}$ M. H. Kelsey, ${ }^{2}$ B. K. Kim, ${ }^{10}$ D. Kong, ${ }^{8}$ Y. F. Lai, ${ }^{1}$ H. B. Lan, ${ }^{1}$ P. F. Lang, ${ }^{1}$ A. Lankford, ${ }^{9}$ F. Li, ${ }_{1}^{1}$ J. Li ${ }^{1}{ }^{1}$ P. Q. Li, ${ }^{1}$ Q. Li, ${ }^{6}$ R. B. Li, ${ }_{1}^{1}$ W. Li ${ }^{1}$ W. D. Li ${ }^{1}{ }^{1}$ W. G. Li, ${ }^{1}$ X. H. Li, ${ }^{1}$ X. N. Li,,${ }^{1}$ S. Z. Lin, ${ }^{1}$ H. M. Liu, ${ }_{1}^{1}$ J. Liu, ${ }^{1}$ J. H. Liu, ${ }^{1}$ Q. Liu, ${ }^{1}$ R. G. Liu, ${ }^{1}$ Y. Liu, ${ }^{1}$ Z. A. Liu, ${ }^{1}$ X. C. Lou, ${ }^{10}$ B. Lowery, ${ }^{10}$ J. G. Lu, ${ }^{1}$ J. Y. Lu, ${ }^{1}$ S. Luo, ${ }^{1}$ Y. Luo, ${ }^{1}$ A. M. Ma, ${ }^{1}$ E. C. Ma, ${ }^{1}$ J. M. Ma, ${ }^{1}$ R. Malchow, ${ }^{4}$ M. Mandelkern, ${ }^{9}$ H. S. Mao, ${ }^{1}$ Z. P. Mao, ${ }^{1}$ X. C. Meng, ${ }^{1}{ }^{H}$ H. L. Ni, ${ }^{1}$ J. Nie, ${ }^{1}$ S. L. Olsen, ${ }^{8}$

J. Oyang, ${ }^{2}$ D. Paluselli, ${ }^{8}$ L. J. Pan, ${ }^{8}$ J. Panetta, ${ }^{2}$ F. Porter ${ }^{2}$ E. Prabhakar, ${ }^{2}$ N. D. Qi ${ }^{1}{ }^{1}$ Y. K. Que, ${ }^{1}$ G. Rong, ${ }^{1}$ M. Schernau, ${ }^{9}$ B. Schmid, ${ }^{9}$ J. Schultz, ${ }^{9}$ Y. Y. Shao, ${ }^{1}$ D. L. Shen, ${ }^{1}$ H. Shen, ${ }^{1}$ X. Y. Shen, ${ }^{1}$ H. Y. Sheng, ${ }^{1}$ H. Z. Shi, ${ }_{1}^{1}$ E. Soderstrom, ${ }^{7}$

X. F. Song, ${ }_{1}^{1}$ J. Standifird ${ }^{10}$ D. Stoker, ${ }^{9}$ F. Sun, ${ }^{1}$ H. S. Sun, ${ }^{1}$ S. J. Sun, ${ }^{1}$ J. Synodinos, ${ }^{7}$ Y. P. Tan, ${ }^{1}$ S. Q. Tang, ${ }^{1}$ W. Toki, ${ }^{4}$ G. L. Tong, ${ }^{1}$ F. Wang, ${ }^{1}$ L. S. Wang, ${ }^{1}$ L. Z. Wang, ${ }^{1}$ M. Wang, ${ }^{1}{ }^{1}$. Wang, ${ }^{1}$ P. L. Wang, ${ }_{1}^{1}$ S. M. Wang, ${ }^{1}$ T. J. Wang, ${ }^{1}$

Y. Y. Wang, ${ }^{1}$ M. Weaver, ${ }^{2}$ C. L. Wei, ${ }^{1}$ W. J. Wisniewski, ${ }^{7}$ D. M. Xi,${ }^{1}{ }^{X}$ X. M. Xia, ${ }^{1}$ P. P. Xie, ${ }^{1}$ D. Z. Xu, ${ }^{1}$ R. S. Xu, ${ }^{1}$ Z. Q. Xu, ${ }^{1}$ S. T. Xue, ${ }^{1}$ J. Yan, ${ }^{1}$ W. G. Yan, ${ }^{1}$ C. M. Yang, ${ }^{1}$ C. Y. Yang, ${ }^{1}$ H. Yang,,${ }^{10}$ W. Yang, ${ }^{4}$ M. H. Ye, ${ }^{1}$ S. Z. Ye, ${ }_{1}^{1}$ K. Young, ${ }^{11}$ C. S. Yu, ${ }^{1}$ C. X. Yu, ${ }^{1}$ Z. Q. Yu, ${ }^{1}$ C. Z. Yuan, ${ }^{1}$ B. Y. Zhang, ${ }^{1}$ C. C. Zhang, ${ }^{1}$ D. H. Zhang, ${ }^{1}$ H. L. Zhang, ${ }^{1}$ J. Zhang, ${ }^{,}$

J. W. Zhang, ${ }^{1}$ L. S. Zhang, ${ }^{1}$ S. Q. Zhang, ${ }^{1}$ Y. Zhang, ${ }^{1}$ Y. Y. Zhang, ${ }_{1}^{1}$ D. X. Zhao, ${ }^{1}$ J. W. Zhao, ${ }_{1}^{1}$ M. Zhao, ${ }^{1}$ W. R. Zhao, ${ }^{1}$ J. P. Zheng, ${ }^{1}$ L. S. Zheng, ${ }^{1}$ Z. P. Zheng, ${ }^{1}$ G. P. Zhou, ${ }^{1}$ H. S. Zhou, ${ }^{1}$ L. Zhou, ${ }^{1}$ X. F. Zhou, ${ }^{1}$ Y. H. Zhou, ${ }^{1}$ Q. M. Zhu, ${ }^{1}$ Y. C. Zhu, ${ }^{1}$ Y. S. Zhu, ${ }^{1}$ and B. A. Zhuang ${ }^{1}$

(BES Collaboration)

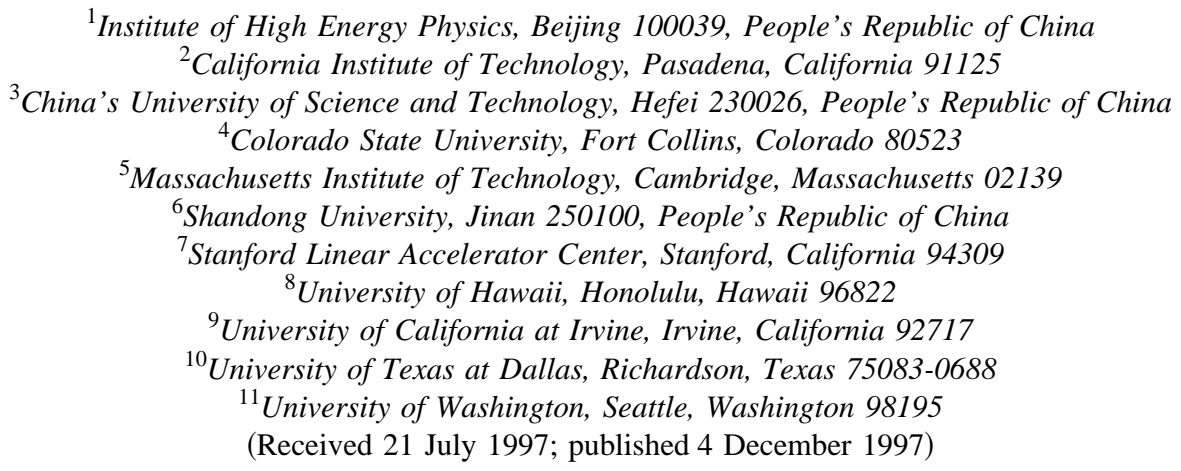

The absolute inclusive branching fraction of $D_{s}^{+} \rightarrow \phi X^{+}$has been measured from data collected by the BES detector at a center-of-mass energy of $4.03 \mathrm{GeV}$, corresponding to an integrated luminosity of $22.3 \mathrm{pb}^{-1}$. At this energy, direct pair production $e^{+} e^{-} \rightarrow D_{s}^{+} D_{s}^{-}$has been observed. We have selected $D_{s}$ candidate events by reconstructing five hadronic decay modes $D_{s}^{+} \rightarrow \phi \pi^{+}, \bar{K}^{0 *} K^{+}, \bar{K}^{0} K^{+}, f_{0} \pi^{+}$and $K^{0} K^{-} \pi^{+} \pi^{+}$and have searched for inclusive $\phi$ 's in the recoiling $D_{s}^{-}$. We observed three recoiling $\phi$ 's in the $166.4 \pm 31.8 D_{s}$

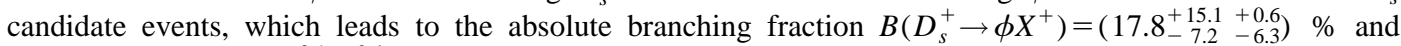
$B\left(D_{s}^{+} \rightarrow \phi \pi^{+}\right)=\left(3.6_{-1.6}^{+3.1}-1.3\right) \quad \%$. [S0556-2821(97)02423-5]

PACS number(s): 13.25.Ft, 14.40.Lb

The main experimental difficulties in charmed particle decays are the problems of overall normalization and the precise determination of charm branching fractions. Previously, we have reported the absolute model-independent branching ratio of $D_{s}^{+} \rightarrow \phi \pi^{+}$[1]. Here we consider the absolute branching fraction of $D_{s}^{+} \rightarrow$ inclusive $\phi$, which contributes toward our understanding of the overall $D_{s}$ branching fraction scale. Moreover the absolute inclusive branching fraction of $D_{s}^{+} \rightarrow \phi X^{+}[2]$ is used in $B_{s}^{0} \bar{B}_{s}^{0}$ oscillation [3] and $B_{s}$

\footnotetext{
*Present address: Northeastern University, Boston, MA.
}

mixing [4] measurements at the CERN $e^{+} e^{-}$LEP and Collider Detector at Fermilab (CDF).

In this paper, we report a direct and model independent measurement of the $D_{s}$ inclusive $\phi$ branching fraction using the BES detector at the Beijing Electron Positron Collider (BEPC). The data were obtained using the BES detector, and correspond to a total integrated luminosity of $22.3 \mathrm{pb}^{-1}$ as determined by large angle Bhabha scattering events at a center-of-mass energy of $4.03 \mathrm{GeV}$. This is just above $e^{+} e^{-} \rightarrow D_{s}^{+} D_{s}^{-}$threshold.

The BES detector is a conventional cylindrical detector, which is described in detail in Ref. [5]. A four-layer central drift chamber (CDC) surrounding the beampipe provides 
trigger information. Outside the CDC, a forty-layer main drift chamber (MDC) provides tracking and energy loss $(d E / d x)$ information on charged tracks over $\sim 85 \%$ of the total solid angle. The momentum resolution is $\sigma_{p} / p=0.017 \sqrt{1+p^{2}}$ ( $p$ in $\mathrm{GeV} / c$ ), and the energy loss $(d E / d x)$ resolution is $\sim 11 \%$ for hadron tracks and $\sim 8.5 \%$ for Bhabha electrons. Scintillation counters that surround the MDC provide time-of-flight (TOF) measurements with resolutions of $\sim 450 \mathrm{ps}$ for hadrons and $\sim 330 \mathrm{ps}$ for Bhabha events. Outside the TOF system, a 12-radiation-length, leadgas barrel shower counter (BSC), operating in limited streamer mode, measures the energies of electrons and photons over $\sim 80 \%$ of the total solid angle. Surrounding the $\mathrm{BSC}$, a solenoidal magnet provides a $0.4 \mathrm{~T}$ magnetic field in the central tracking region of the detector. Three doublelayer muon counters (MUC) instrument the magnet flux return and serve to identify muons of momentum greater than $0.5 \mathrm{GeV} / c$. They cover $\sim 68 \%$ of the total solid angle with longitudinal (transverse) spatial resolution of $5 \mathrm{~cm}(3 \mathrm{~cm})$.

In this experiment, the $D_{s}$ signal has been detected via five hadronic decay modes: $D_{s}^{+} \rightarrow \phi \pi^{+}, \bar{K}^{0 *} K^{+}, \bar{K}^{0} K^{+}$, $f_{0} \pi^{+}$or $K^{0} K^{-} \pi^{+} \pi^{+}$. The subresonances are detected by the decays $\phi \rightarrow K^{+} K^{-}, \bar{K}^{0 *} \rightarrow K^{-} \pi^{+}, \bar{K}^{0} \rightarrow K_{S}^{0} \rightarrow \pi^{+} \pi^{-}$, and $f_{0} \rightarrow \pi^{+} \pi^{-}$. Each candidate was formed using wellreconstructed tracks. Each track's closest approach to the origin was required to be smaller than $1.2 \mathrm{~cm}$ in the $x y$ plane and $15 \mathrm{~cm}$ in the $z$ direction. For the $f_{0} \pi$ mode, the vertexing requirements were tightened to $0.65 \mathrm{~cm}$ in the $x y$ plane and $9 \mathrm{~cm}$ in the $z$ direction due to larger backgrounds. Vertexing requirements were not applied to candidate pions from $K_{S}^{0}$ decay. The proper decay time of $K_{S}^{0}$ candidates was required to be between 0.01 and $0.33 \mathrm{~ns}$. Additionally in the $K^{0} K^{-} \pi^{+} \pi^{+}$mode, the $K_{S}^{0}$ vertex was required to have the difference between the $z$ coordinates of the two tracks to be within $4 \mathrm{~cm}$, and the $x y$ alignment of the parent momentum with the line from the interaction point to the $K_{S}^{0}$ vertex was required to have a confidence level $>5 \%$. A fiducial requirement, $|\cos \theta|<0.85$ was used for charged tracks. Both TOF and $d E / d x$ systems were used to reduce background from random combinations of different particles. Time-of-flight and $d E / d x$ information associated with each track was required to be consistent with the assigned mass interpretation with a confidence level $>1 \%$. Kaon candidates were required to have $\chi^{2}(K)<\chi^{2}(\pi)$. Pion candidates were required to have $\chi^{2}(\pi)<\chi^{2}(K)$ and additionally $\chi^{2}(\pi)<\chi^{2}(e)$ for $f_{0} \pi^{+}$candidates. Candidates for $\phi, \bar{K}^{0 *}$, $\bar{K}^{0}$, and $f_{0}$ were required to be within $18,60,20$, and 30 $\mathrm{MeV} / \mathrm{c}^{2}$, respectively, of the nominal mass.

Additional background rejection was obtained with helicity angle cuts for the $\phi$ and $\bar{K}^{0 *}$. We required $\left|\cos \theta_{K}\right|>0.25$ in the $\phi$ rest frame for the $\phi \pi$ mode and $\left|\cos \theta_{K}\right|>0.4$ in the $\bar{K}^{0 *}$ rest frame for the $\bar{K}^{0 *} K$ mode.

The production of $D_{s}$ has a $\sin ^{2} \theta$ distribution with respect to the beam direction. In single tag modes with large backgrounds, the signal-to-noise ratio can be improved by only using tags with small values of $\cos \theta$. We required $\left|\cos \theta_{D_{s}}\right|<0.7$ for the $\bar{K}^{0 *} K^{+}$mode, and $\left|\cos \theta_{D_{s}}\right|<0.85$ for both the $f_{0} \pi^{+}$and the $\bar{K}^{0} K^{+}$modes.
Candidates satisfying these criteria were subjected to a one-constraint (1C) kinematic fit to the beam energy. Those having a fit confidence level $>1 \%$ for $\phi \pi^{+}, \bar{K}^{0 *} K^{+}$and $\bar{K}^{0} K^{+},>5 \%$ for $K^{0} K^{-} \pi^{+} \pi^{+}$, and $>10 \%$ for $f_{0} \pi^{+}$were retained. The unbinned maximum likelihood fit to each single tag plot [Figs. 1(a)-1(e)] gave the number of single tag events $\left(N_{\text {sngl }}^{i}\right)$, and fitting to Fig. 1(f) gave a total of $166.4 \pm 31.8 D_{s}$ single tags above background.

Double tagged $D_{s}$ events were obtained by reconstructing a $\phi \rightarrow K^{+} K^{-}$recoiling against one of the five $D_{s}$ single tag modes. Recoiling $\phi$ 's were selected with the same track and particle identification requirements described earlier for the $\phi \pi$ single tag mode. $\phi$ candidates were required to be within $18 \mathrm{MeV} / \mathrm{c}^{2}$ of the $\phi$ mass (3 times the resolution for the reconstructed $\phi$ mass). A total of 3 double tag events (Fig. 2) were found, and the characteristics of these events are summarized in Table I.

A direct measurement of $B_{\phi X}$ is obtained from the number of single tag events $\left(N_{\text {sngl }}\right)$, the number of double tag events $\left(N_{\mathrm{dbl}}\right)$, and the inclusive $\phi$ efficiency $\left(\epsilon_{\mathrm{dbl}}\right)$ as follows:

$$
B_{\phi X}=\frac{N_{\mathrm{dbl}}}{N_{\mathrm{sngl}} \times \epsilon_{\mathrm{dbl}} \times B\left(\phi \rightarrow K^{+} K^{-}\right)} .
$$

The inclusive $\phi$ double tag efficiency, $\epsilon_{\mathrm{dbl}}$, was determined using Monte Carlo simulation for each of the five modes (Table II).

A $D_{s}$ tag side band method was used to estimate the background in the double tag sample. As consistency checks three different methods were used in the background estimation: recoil $\phi$ side band method, tag subresonance side band method, and Monte Carlo background studies.

The $D_{s}$ tag side band regions (Fig. 2) were defined from $1.79-1.957 \mathrm{GeV} / c^{2}$ and from $1.981-2.105 \mathrm{GeV} / c^{2}$. One $\phi$ was found recoiling from a sideband of the $\bar{K}^{0 *} K^{+}$tag. No background event was detected in the $\phi \pi^{+}$side band region. For both $\phi \pi^{+}$and $\bar{K}^{0 *} K^{+}$channels, we have normalized the tag side bands by the ratio of single tag events in the signal and side band regions. Poisson errors for a single event in the $\bar{K}^{0 *} K^{+}$side band region implies an estimated background of $0.13_{-0.04}^{+0.30}$. In order to express the uncertainty of the $\phi \pi$ mode background, we have used the $84.1 \%$ confidence level upper limit for zero events giving $0.0_{-0.0}^{+0.26}$.

There is no background event from the the recoil $\phi$ mass side band (Fig. 2) method.

All analyses were repeated using the side bands of the tag subresonances, $0.965-1.001$ and $1.037-1.073 \mathrm{GeV} / \mathrm{c}^{2}$ for $\phi$, $0.712-0.832$ and $0.952-1.072 \mathrm{GeV} / c^{2}$ for $\bar{K}^{* 0}, 0.4376-$ 0.4776 and $0.5176-0.5576 \mathrm{GeV} / c^{2}$ for $\bar{K}^{0}, 0.89-0.95$ and $1.01-1.07 \mathrm{GeV} / c^{2}$ for $f_{0}$. No event passed the selection criteria, leading to an estimate of zero background events in our double tag sample.

Finally, large Monte Carlo samples of $D^{*+} D^{-}, D^{* 0} D^{0}$, $D^{*+} D^{*-}$, and $D^{* 0} D^{* 0}$ were used to estimate backgrounds from these sources. These samples correspond to 6.4, 5.9, 5.3 and 5.0 times the real data sample respectively. All Monte Carlo background events in the double tag signal region were rejected. 

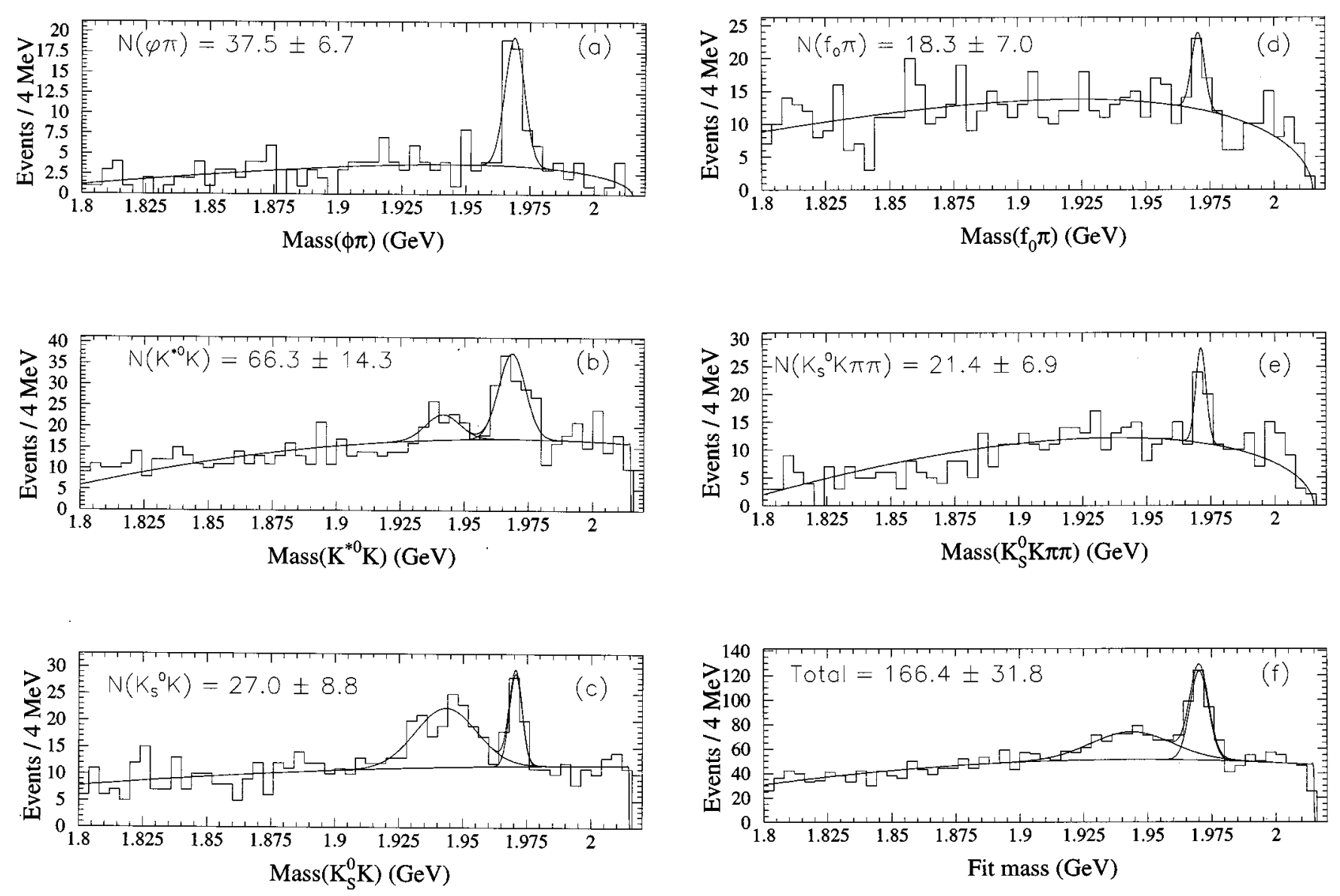

FIG. 1. Kinematically fit mass of $D_{s}$ candidates. The curves are the result of unbinned fits to the data.

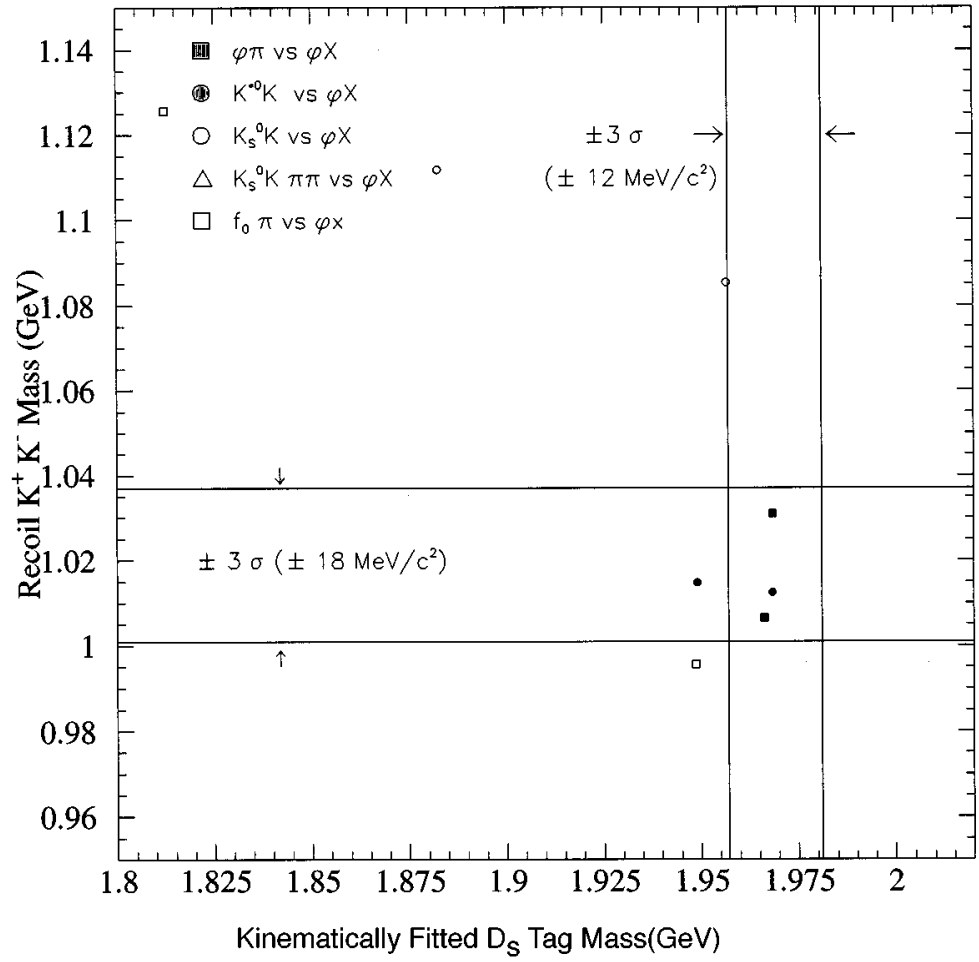

FIG. 2. Double tag candidates. 
TABLE I. Properties of the double tag candidates.

\begin{tabular}{lccc}
\hline \hline Event & 1 & 2 & 3 \\
\hline Tagging $D_{s}$ Decay & $\phi \pi^{+}$ & $\phi \pi^{+}$ & $\bar{K}^{0 *} K^{+}$ \\
Subsystem mass $\left(\mathrm{GeV} / c^{2}\right)$ & 1.0090 & 1.0229 & 0.8345 \\
$D_{s}$ invariant mass $\left(\mathrm{GeV} / c^{2}\right)$ & 1.9723 & 1.9694 & 1.9678 \\
$1 \mathrm{C} D_{s}$ fitmass $\left(\mathrm{GeV} / c^{2}\right)$ & 1.9662 & 1.9686 & 1.9684 \\
Recoiled $\phi$ mass $\left(\mathrm{GeV} / c^{2}\right)$ & 1.0068 & 1.0306 & 1.0125 \\
Number of visible charged tracks & 6 & 5 & 6 \\
Number of isolated showers & 5 & 3 & 1 \\
\hline \hline
\end{tabular}

Since there are no signal events in the $\bar{K}^{0} K^{+}, f_{0} \pi^{+}$, and $K^{0} K^{-} \pi^{+} \pi^{+}$modes, the background estimates do not affect the shape of the likelihood function.

Combining all four different methods gives estimated backgrounds of $0.0_{-0.0}^{+0.26}$ events for the $\phi \pi^{+}$mode and $0.13_{-0.04}^{+0.30}$ events for the $\bar{K}^{0 *} K$ mode. The background uncertainties contribute ${ }_{-35.0}^{+1.0} \% \times B_{\phi X}$ to the systematic error for the branching fraction $B_{\phi X}$.

The value of $B_{\phi X}$ is obtained using a maximum likelihood method. The likelihood function,

$$
L^{i}\left(B_{\phi X}, N_{\mathrm{sngl}}^{i} ; \epsilon_{\mathrm{dbl}}^{i}, N_{\mathrm{dbl}}^{i}, N_{\mathrm{bg}}^{i}\right)
$$

is constructed from Eq. (1) using a Poisson distribution to describe the number of double tag events and a Gaussian distribution to describe the single tag sample:

$$
L\left(B_{\phi X}\right)=\prod_{i} L_{\operatorname{mar}}^{i}\left(B_{\phi X}\right)
$$

where $i$ refers to the single tag mode. The marginalized likelihood function for each of the five different $D_{s}$ modes is obtained by integrating out the single tag uncertainty:

$$
\begin{aligned}
L_{\mathrm{mar}}^{i}\left(B_{\phi X}\right)= & \int d \widetilde{N}_{\mathrm{sngl}}^{i} L^{i}\left(B_{\phi X}, \widetilde{N}_{\mathrm{sngl}}^{i}\right) \\
& \times \frac{\exp \left[-\frac{1}{2}\left(\frac{N_{\mathrm{sngl}}^{i}-\widetilde{N}_{\mathrm{sngl}}^{i}}{\delta N_{\mathrm{sngl}}^{i}}\right)^{2}\right]}{\sqrt{2 \pi} \delta N_{\mathrm{sngl}}^{i}} .
\end{aligned}
$$

The likelihood function $L^{i}$ is given by

$$
L^{i}=\frac{A_{i}^{N_{\mathrm{dbl}}^{i}}}{N_{\mathrm{dbl}}^{i} !} e^{-A_{i}},
$$

TABLE II. Result of the measurement.

\begin{tabular}{lcccc}
\hline \hline Decay mode & $N_{\text {sngl }}^{i}$ & $N_{\mathrm{dbl}}^{i}$ & $\epsilon_{\mathrm{db} 1}^{i}$ & $N_{b g}^{i}$ \\
\hline$\phi \pi^{+}$ & $37.5 \pm 6.7$ & 2 & $0.202 \pm 0.004$ & $0.0_{-0.0}^{+0.26}$ \\
$\bar{K}^{0 *} K^{+}$ & $66.3 \pm 14.3$ & 1 & $0.200 \pm 0.005$ & $0.13_{-0.04}^{+0.30}$ \\
$\bar{K}^{0} K^{+}$ & $27.0 \pm 8.8$ & 0 & $0.190 \pm 0.004$ & N/A \\
$f_{0} \pi^{+}$ & $18.3 \pm 7.0$ & 0 & $0.180 \pm 0.005$ & N/A \\
$K^{0} K^{-} \pi^{+} \pi^{+}$ & $21.4 \pm 6.9$ & 0 & $0.181 \pm 0.007$ & N/A \\
\hline \hline
\end{tabular}

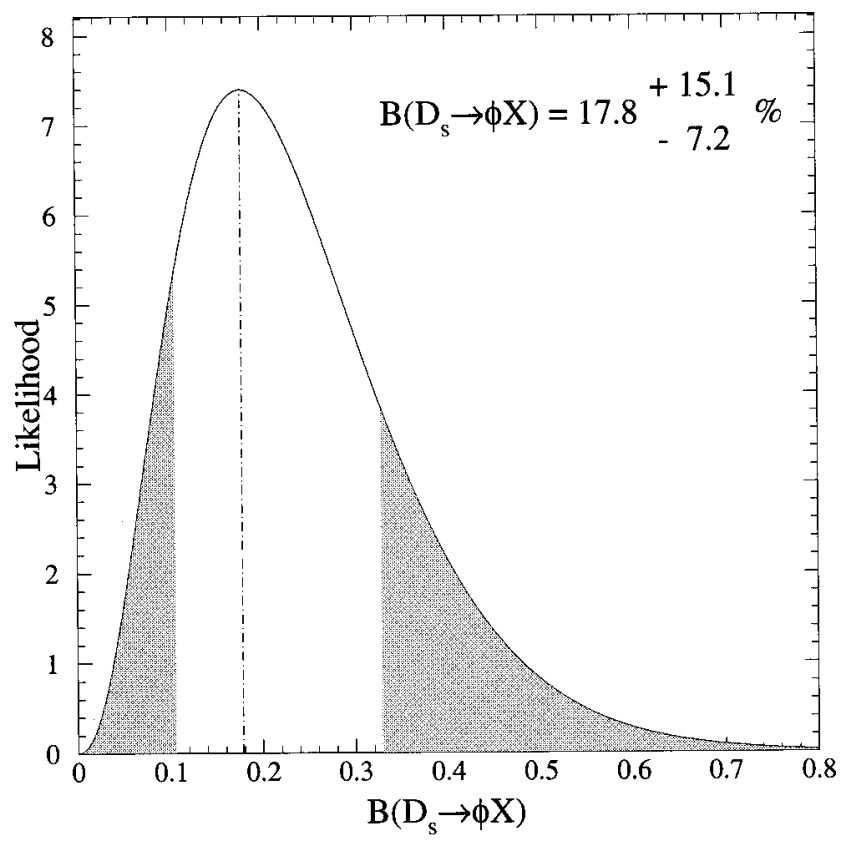

FIG. 3. The variation of the normalized likelihood function with respect to $B_{\phi X}$; the unshaded area under the curve denotes the $68 \%$ confidence interval.

where

$$
A_{i} \equiv B_{\phi X} N_{\mathrm{sngl}}^{i} \epsilon_{\mathrm{dbl}}^{i} B\left(\phi \rightarrow K^{+} K^{-}\right)+N_{b g}^{i}
$$

The value of the likelihood function, $L\left(B_{\phi X}\right)$, is shown in Fig. 3. The maximum likelihood solution is $B_{\phi X}=\left(17.8_{-7.2}^{+15.1}\right) \%$, where the statistical errors are obtained by integrating the function; the area under the curve between the peak value and $-1 \sigma(+1 \sigma)$ corresponds to $68 \%$ of the total area below(above) the peak position.

Several systematic uncertainties affect this measurement. The inclusive $\phi$ efficiency, $\epsilon_{\mathrm{dbl}}$, introduces a systematic error for $B_{\phi X}$ of $2.4 \% \times B_{\phi X}$. The choice of a background functional form and fit interval for the single tag sample introduces a $2.0 \% \times B_{\phi X}$ uncertainty. Finally, the double tag background estimate is responsible for $\mathrm{a}_{-35.0}^{+1.0} \% \times B_{\phi X}$ uncertainty. After combining the systematic errors in quadrature, the final result for $B_{\phi X}$ is

TABLE III. Inclusive $\phi$ decay modes of $D_{s}$ (PDG 1996).

\begin{tabular}{lcc}
\hline \hline Decay mode & Branching fraction $(\%)$ & $\Gamma_{i} / \Gamma_{\phi \pi}$ \\
\hline$D_{s}^{+} \rightarrow \phi e^{+} \nu$ & $1.9 \pm 0.5$ & $0.54 \pm 0.05$ \\
$D_{s}^{+} \rightarrow \phi \mu^{+} \nu$ & $1.9 \pm 0.5$ & $0.54 \pm 0.05$ \\
$D_{s}^{+} \rightarrow \phi \pi^{+}$ & $3.6 \pm 0.9$ & 1.00 \\
$D_{s}^{+} \rightarrow \phi \pi^{+} \pi^{0}$ & $9 \pm 5$ & $2.4 \pm 1.0 \pm 0.5$ \\
$D_{s}^{+} \rightarrow \phi \pi^{+} \pi^{+} \pi^{-}$ & $1.8 \pm 0.6$ & $0.51 \pm 0.12$ \\
$D_{s}^{+} \rightarrow \phi K^{+}$ & $<0.05$ & $<0.071$ \\
Total & $18.2 \pm 5.2$ & $5.0 \pm 1.0 \pm 0.5$ \\
\hline \hline
\end{tabular}




$$
B_{\phi X}=\left(17.8_{-7.2}^{+15.1+0.6}-6.3\right) \% .
$$

This is a direct measurement of the $D_{s}$ inclusive $\phi$ branching fraction that is model-independent. The present world average value from indirect or model-dependent procedures is $B_{\phi X}=(18.2 \pm 5.2) \%$ (Table III).

LEP experiments [3] and CDF [4] have used a theoretically inspired method to estimate $B_{\phi X}$ from $B_{\phi \pi}$. The theoretical $D_{s}^{+}$branching fractions are evaluated from the BSW model [6], giving $B\left(D_{s}^{+} \rightarrow \phi X^{+}\right)=(4.84 \pm 0.51) b_{s}$ [7], where $b_{s}$ is the measured branching fraction of $D_{s}^{+} \rightarrow \phi \pi^{+}$. Using the Particle Data Group (PDG) (1996) [8] value yields $B_{\phi X}=(17.4 \pm 4.7) \%$.

A measurement of $B_{\phi \pi}$ can be obtained from $B_{\phi X}$ using the sum of exclusive measurements shown in the Table III under the assumption that no significant decays of the $D_{s}$ to $\phi$ remain unmeasured. Scaling $B_{\phi X}$ by the sum of the world average values of $\Gamma_{i} / \Gamma_{\phi \pi}$ gives $B_{\phi \pi}=\left(3.6_{-1.6-1.3}^{+3.1+0.4}\right) \%$. This value is consistent with the previous BES result $B_{\phi \pi}=\left(3.9_{-1.9-1.1}^{+5.1+1.8}\right) \%[1]$.

We would like to thank the staffs of the BEPC accelerator and the Computing Center at the Institute of High Energy Physics (Beijing). This work was supported in part by the National Natural Science Foundation of China under Contract No. 19290400 and the Chinese Academy of Sciences under Contract No. KJ85 (IHEP); by the Department of Energy under Contract Nos. DE-FG03-92ER40701 (Caltech), DE-FG03-93ER40788 (Colorado State University), DEAC02-76ER03069 (MIT), DE-AC03-76SF00515 (SLAC), DE-FG03-91ER40679 (UC Irvine), DE-FG03-94ER40833 (University of Hawaii), DE-FG03-95ER40925 (UT Dallas); by the U.S. National Science Foundation, Grant No. PHY9203212 (University of Washington).
[1] J. Z. Bai et al., Phys. Rev. D 52, 3781 (1995).

[2] Throughout the paper, reference to a particular charge configuration implies reference to the charge conjugate configuration as well.

[3] X. C. Lou, in Proceeding of the Workshop on B Physics at Hadron Accelerators, Snowmass, Colorado, 1993, edited by C. Shekhar Mishra and P. McBride (Fermilab, Batavia, 1994).

[4] John E. Skarha and A. Barry Wicklund, in Proceeding of the
Workshop on B Physics at Hadron Accelerators [3].

[5] J. Z. Bai et al., Nucl. Instrum. Methods Phys. Res. A 344, 319 (1994); J. Z. Bai et al., Phys. Rev. Lett. 69, 3021 (1992).

[6] M. Bauer, B. Stech, and M. Wirbel, Z. Phys. C 34, 103 (1987).

[7] P. Roudeau and A. Stocchi (unpublished).

[8] Particle Data Group, R. Barnett et al., Review of Particle Physics, Phys. Rev. D 54, 1 (1996). 